\title{
DIRECTIVE RADIO TRANSMISSION ON A WAVE LENGTH OF 10 METERS.
}

\author{
By Francis W. Dunmore and Francis H. Engel.
}

\begin{abstract}
.
Interference between different radio transmitting stations can be reduced by the use of markedly directional antennas for transmitting and receiving and by the use of short wave lengths not at present employed. For broadcasting, such as the transmission of market reports or music, directional antennas are not suitable for transmitting, but can be used for reception. For point-to-point communication-that is, from one transmitting station to one receiving station-directional antennas can advantageously be employed for both transmission and reception. Radio communication has so far been carried on, with few exceptions, on wave lengths of not less than $200 \mathrm{~m}$. The use of much shorter waves, such as ro meters, with the accompanying reduction of interference, has not been adopted to any extent. Experiments recently
\end{abstract} made by Marconi and others have shown them to be practicable.

This paper describes a series of experiments in radiotelegraph and radiotelephone transmission on a wave length of $10 \mathrm{~m}$, using at the transmitting station a reflector consisting of short, vertical wires arranged as elements of a parabolic cylinder. The system described, therefore, employs two means of reducing interference. In the work described in this paper a $5^{\mathrm{o}-w a t t}$ electron-tube generating set was employed. The capacity between the elements of the tube, together with the grid and plate coupling coils, formed the generating circuit. Waves were radiated from the generating set by two short, vertical, multiple-wire conductors, one placed above and one below the generating set. A coupling coil was connected between the two vertical conductors, and the generating set thus coupled to the system. The generating set with the vertical conductors was suspended in the focal axis of the parabolic cylinder. Each wire of the reflector was tuned separately to Io $\mathrm{m}$ by adjusting its length. With all adjustments of the reflector correctly made, good directional transmission was obtained. At least 75 per cent of the radiated power was confined to an angle of $40^{\circ}$.

Studies were made of the effect of removing the wires near the center of the reflector, of removing alternate wires in all parts of the reflector, of detuning the wires, and of varying the aperture of the reflector. For studying the radiation characteristics of the system, a single-turn loop-receiving antenna was used with a small condenser, thermocouple, and microammeter. Measurements of received current were made with this receiving apparatus while the transmitting reflector was rotated. Radiation characteristics for various adjustments of the reflector are shown by means of polar curves.

The paper gives the constructional details of the apparatus employed, so that any person interested can duplicate the apparatus used and the results obtained, and can continue in this line of investigation.

CONTENTS.

I. Introduction.

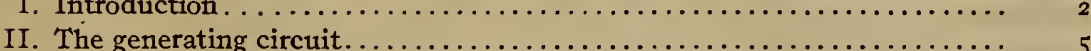

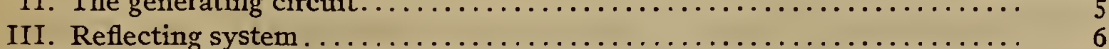

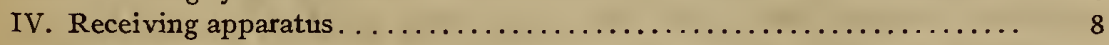

V. Results ................................... 9 


\section{INTRODUCTION.}

One of the most important problems of radio communication is the interference between different transmitting stations. The radio waves which are transmitting radiotelephone messages usually occupy a broader band of wave lengths than the waves which are transmitting radiotelegraph continuous-wave code signals. There is a definite and not very large limit to the number of radiotelephone transmitting stations of any considerable power which can operate in a given locality without serious interference, employing the usual types of antennas and transmitting apparatus. In the past radio communication has been almost entirely carried on by using wave lengths greater than $200 \mathrm{~m}$ and nondirective antennas which radiated about equally well in every direction. At short wave lengths a more narrow band of wave lengths is required to transmit a given sound in radiotelephony than is required at long wave lengths.

At the present time many radiotelephone transmitting stations are transmitting music and other entertainment broadcast for reception by a large number of receiving stations located in all directions from the transmitting station. For such broadcast transmission directive antennas are not suitable, but the use of directional antennas for the reception of broadcasted radiotelephone messages offers a means of reducing interference difficulties at a receiving station.

Directive antennas for transmission are, however, desirable for "point-to-point" communication; that is, communication from one transmitting station to one receiving station. Transoceanic and much of ship radio traffic are practically all point-to-point communication. There are many cases in which communication is desired between points not easily accessible, so that radio communication is the only practicable means. The use of a method of directive transmission greatly reduces the interference which such communication will cause. There are some new kinds of point-to-point radio communication which are now being developed, such as the transmission of photographs by radio and the remote control of mechanisms by radio, all of which applications can advantageously be carried on by directive short-wave transmission.

The enormous increase in the use of radiotelegraphy and radiotelephony during the past two years has created a demand for apparatus capable of being operated with a minimum of inter- 
ference. Directive transmission should make possible such communications, with a minimum amount of power as well as with the least interference. Directive transmission on very short wave lengths (below $20 \mathrm{~m}$ ) seems to offer the most promising solution at present in sight.

In recent papers by Franklin, ${ }^{1}$ Marconi, ${ }^{2}$ and others interesting and valuable data have been given on directive radio transmission using wave lengths below $20 \mathrm{~m}$.

The present paper describes a brief series of experiments, employing a wave length of $10 \mathrm{~m}$, the results of which confirm the work of these investigators. An effort is made not only to present the results of the experiments, but also to give details regarding the apparatus and circuits used, including circuit constants, so that anyone may duplicate the results obtained by the authors or continue in this line of investigation.

The experiments here described make use of directive antennas for transmission. It is also possible to use antennas of marked directional characteristics for reception, and thus to reduce interference in reception caused by undesired transmitting stations. This is the next logical step in the development of a directional system of communication. Since the waves are reflected and radiated in one direction, a less powerful generating set is required to cover a given distance than when a nondirective antenna is used.

Another serious difficulty in radio communication is due to "strays," which are stray waves caused by atmospheric electric disturbances. These disturbances are not infrequently so severe, particularly during the summer months, as to make satisfactory communication impossible. It is general experience that strays are less severe on short wave lengths than on long wave lengths, and at such short wave lengths as $10 \mathrm{~m}$ it has been found that strong strays are not ordinarily encountered. Strays of some kinds come from a particular direction and can be practically eliminated by the use in reception of an antenna of marked directional characteristics. More serious difficulties are experienced from strays when a large antenna is used; a very small antenna is used for such short wave lengths as Io $\mathrm{m}$. For several reasons, therefore, a system of communication on $10 \mathrm{~m}$, which employs directional antennas, greatly reduces the difficulties due to strays.

\footnotetext{
1 C. S. Franklin, Short Wave Directional Wireless Telegraphy, Wireless World and Radio Review, 10, pp. $219-225$, May $20,1922$.

2 Radio Telegraphy, Proceedings of the Institute of Radio Engineers, 10, pp. 215-238, August, I922.
} 
A brief elementary discussion of strays and of directional antennas, including coil antennas for direction finding, may be found in The Principles Underlying Radio Communication, Signal Corps Radio Communication Pamphlet No. 40.

The possibilities of short-wave transmission, both directive and nondirective, are so great that the value of further research along these lines is apparent. It is hoped that the results of this paper,

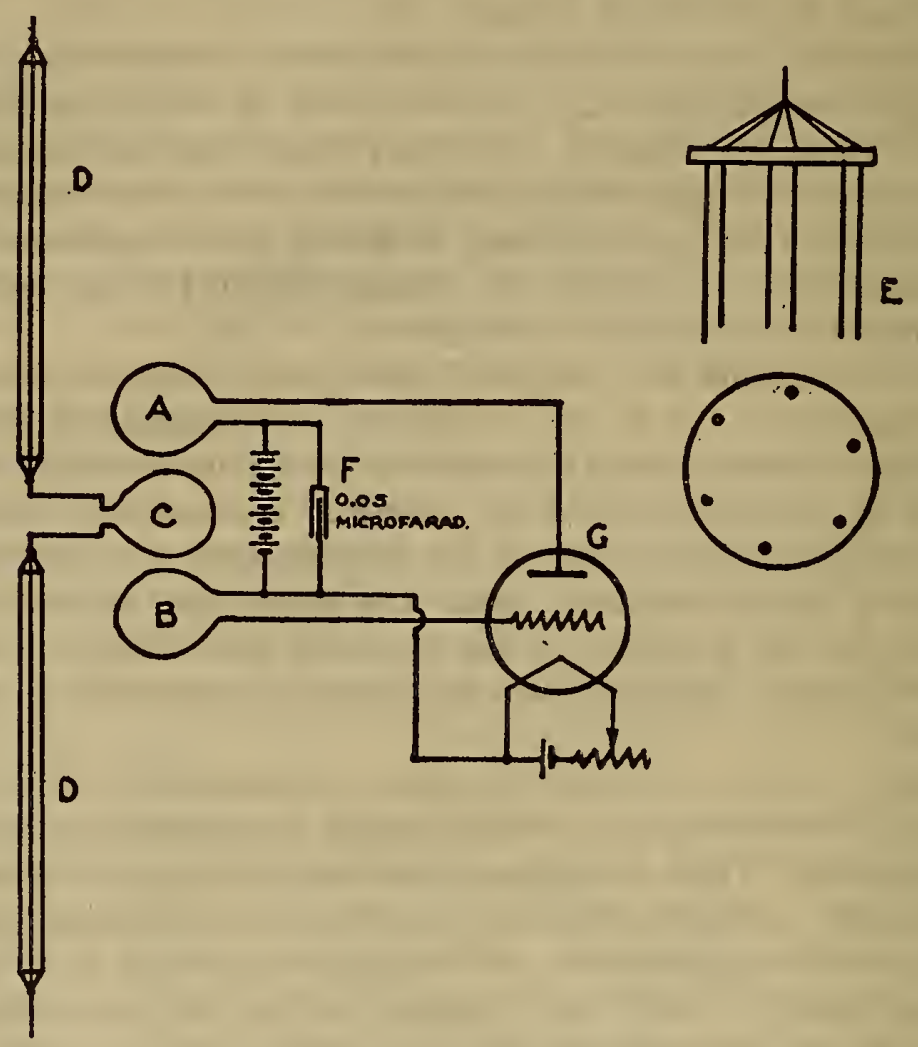

FIG. I.-Circuit of Io-meter generating set.

while incomplete, may encourage further investigation along this line.

The problem of the generation and directive radiation of waves of the order of ro $\mathrm{m}$ resolves itself into (I) the development of a ro $\mathrm{m}$ electron-tube generator (of suitable power), (2) the development of an efficient directive reflecting system for radio waves of this wave length, and (3) the development of ro $\mathrm{m}$ receiving apparatus. 


\section{THE GENERATING CIRCUIT.}

Various three-electrode tubes of commercially available types were tested, a 50-watt tube of the coated-filament type being finally selected for these experiments, since it functioned at higher frequencies than any other tube tested. The frequency at which this tube was operated during these tests was $30,000 \mathrm{kc} / \mathrm{s}$ (IO m).

The circuit is shown in Figure I. It is a Hartley circuit. Coil $A$ consists of a single turn $17 \mathrm{~cm}$ in diameter for plate coupling, and coil $B$ is a similar coil for grid coupling. The capacity between the elements of the tube, together with these coils, forms the oscillatory circuit. It is this internal capacity which determines the upper limit of the frequencies obtainable with a given tube.

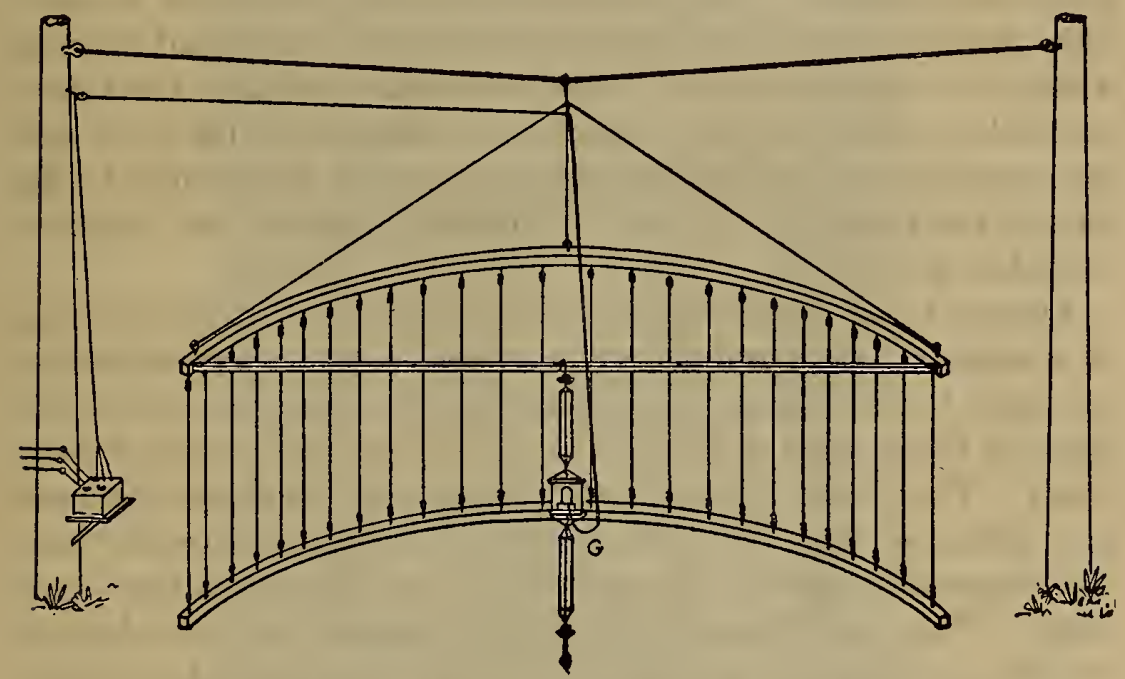

FIG. 2.-Parabolic reflector for short-wave directive transmission.

The radiating system (the antenna) $D$ (Fig. I) is coupled to the generating circuit by means of coil $C$, which is similar to coils $A$ and $B$. The antenna $D$ consists of two sets of vertical wires connected by means of coil $C$. Each set consists of six parallel wires arranged in a circle, as shown at $E$, Figure $\mathbf{~ . ~ T h e s e ~ w i r e s ~}$ were spaced about $3 \mathrm{~cm}$ apart and were $1.8 \mathrm{~m}$ in length.

Figure 3 is a view of the generating set showing grid, plate, and antenna coupling coils. The plate and grid coils are supported on each side of the tube by the tube socket, thus reducing the length of connections to a minimum. The radio-frequency by-pass condenser $F$ (Figs. I and 3 ) is mounted directly under the tube 
socket for the same reason. It is obvious that all leads should be kept as short as possible, since at these high frequencies the leads may form an appreciable part of the total inductance in the circuit. The generating set shown in Figure 3 was made for experimental purposes and is not mechanically suited for permanent use. For practical use the coils $A$ and $B$ should be rigidly supported, since any relative movement between them causes a change in the frequency of the generated current.

\section{REFLECTING SYSTEM.}

There are several ways of obtaining directive transmission, but the most effective method for short wave lengths consists of the use of a reflector of proper design in the form of a section of a parabolic cylinder. The wave from this form of reflector is somewhat similar to a parallel beam of light which has passed through a slit in an opaque screen. From theoretical considerations for a parabolic cylinder having a line source situated in the focal axis the reflected rays will all be parallel and will be parallel to the axis of the parabolic cylinder. This ideal result is only approximated in practice.

Figures 2 and 4 illustrate the reflector used. It is in the shape of a segment of a parabolic cylinder and is made by suspending 40 wires from a frame constructed in the form of a parabola. Each of these wires is tuned to ro $\mathrm{m}$ and spaced $30.47 \mathrm{~cm}$ (I foot) apart. The frame is suspended from a rope stretched between two poles, so that the reflector may be rotated through $360^{\circ}$. The suspended wires are insulated from the frame and from each other. The focal distance was made one-quarter of a wave length; that is, $2.5 \mathrm{~m}$ ( 8 feet 2.4 inches). With this distance determined, the parabolic frame may be constructed. It is important to have a frame of this shape in order to maintain the proper phase relations and to obtain maximum reflection. The reason for this will be made clear by reference to Figure 5 , which may be considered a simplified plan view of Figure 2. The filament rheostat and ammeters in the filament and plate supply circuits are mounted in a small unit shown at the left in Figures 2 and 4.

In Figure 5 the $10 \mathrm{~m}$ electron-tube generator is shown at the focus $G$ at a distance of one-fourth of a wave length from the vertex $B$. The curve $C B A$ is parabolic. Each wire along $C B A$ reradiates the energy received from $G$ by virtue of the fact that it 
Scientific Papers of the Bureau of Standards, Vol. 19

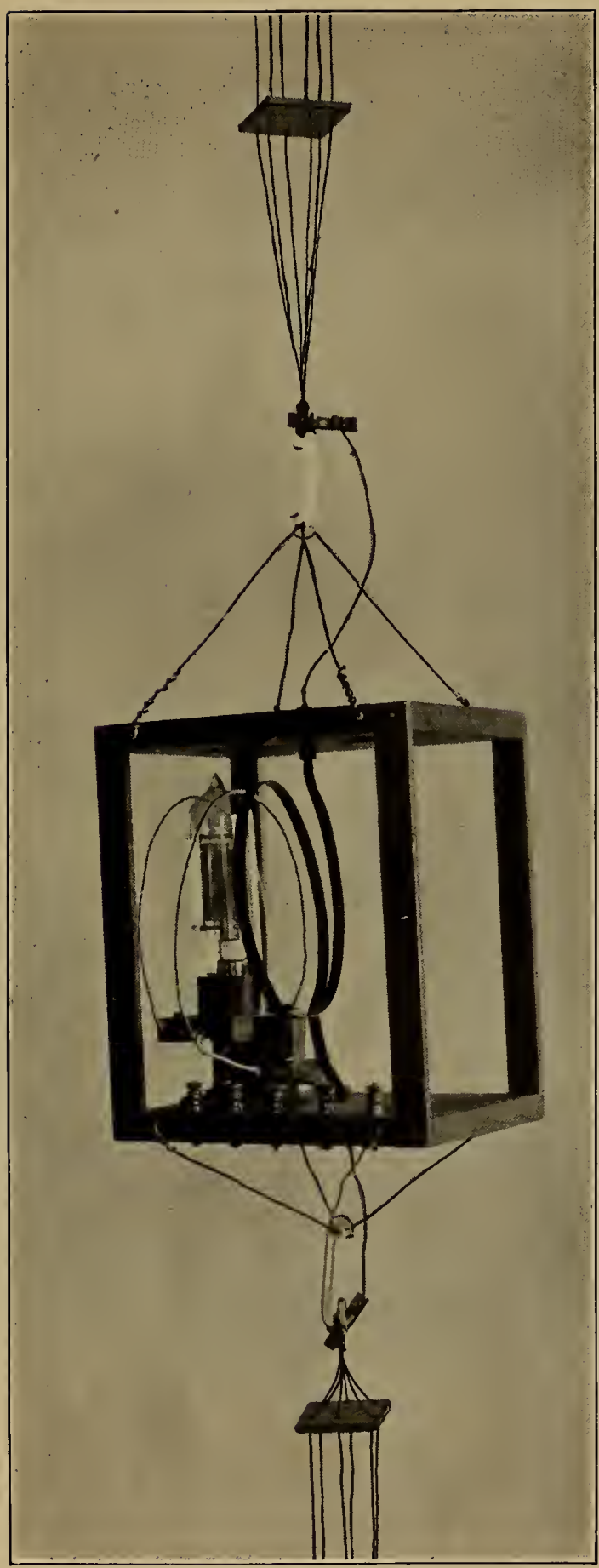

FIG. 3.-Assembled IO-meter generating set showing method of coupling to antenna. 
Scientific Papers of the Bureau of Standards, Vol. 19

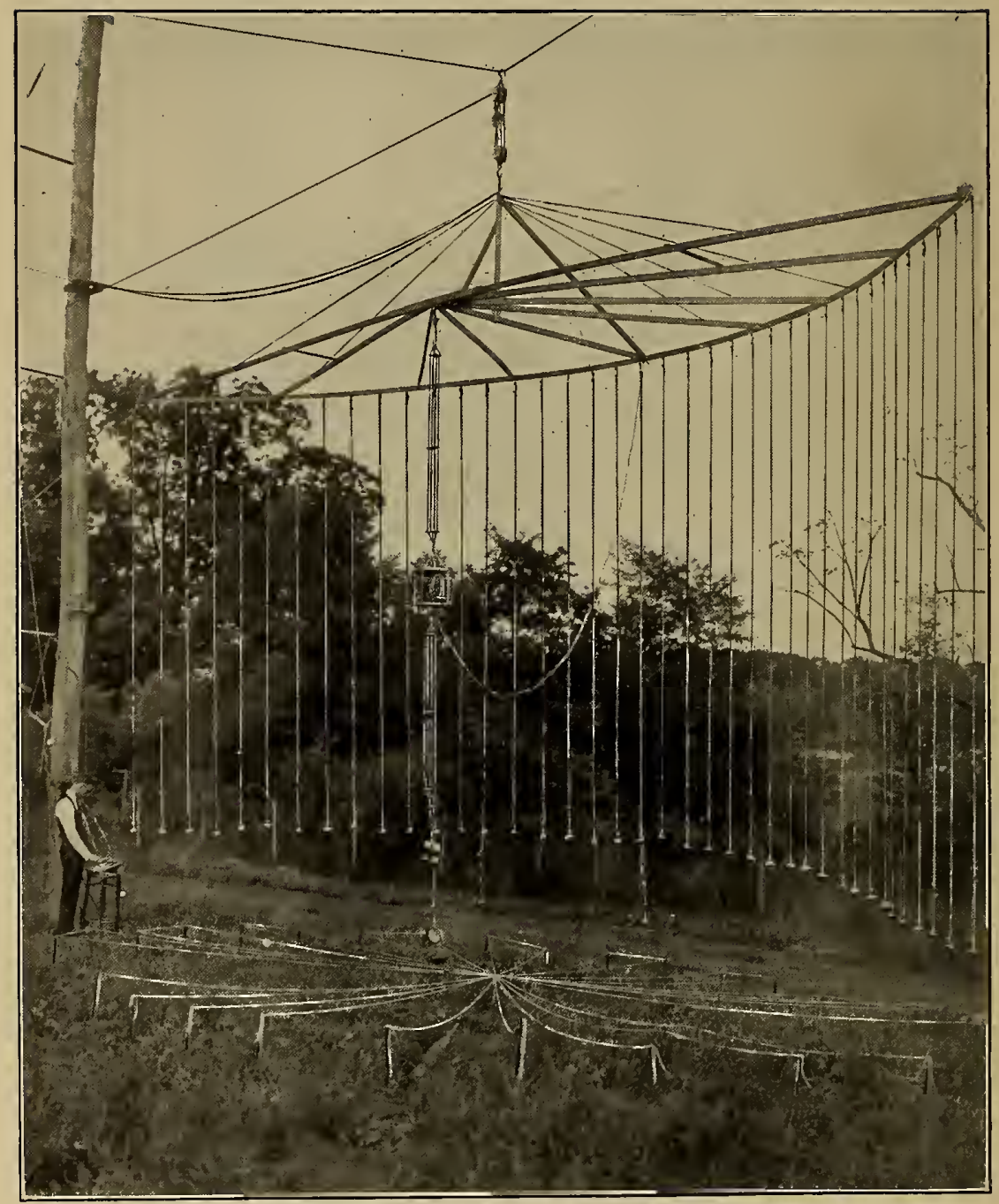

FIG. 4.-Parabolic reflector for a wave length of ro meters. 
is tuned to $G$. Since any distance $G K O$ or $G P L$ is equal to $G B+B G$, it is evident that the reradiation from all the wires along $C B A$ will reach the aperture $C A$ in phase with each other. It is evident, therefore, that reenforcement takes place in the direction $G M$ and interference in the direction $B T$. Except for leakage, as shown at $G W$, practically all the energy is reflected

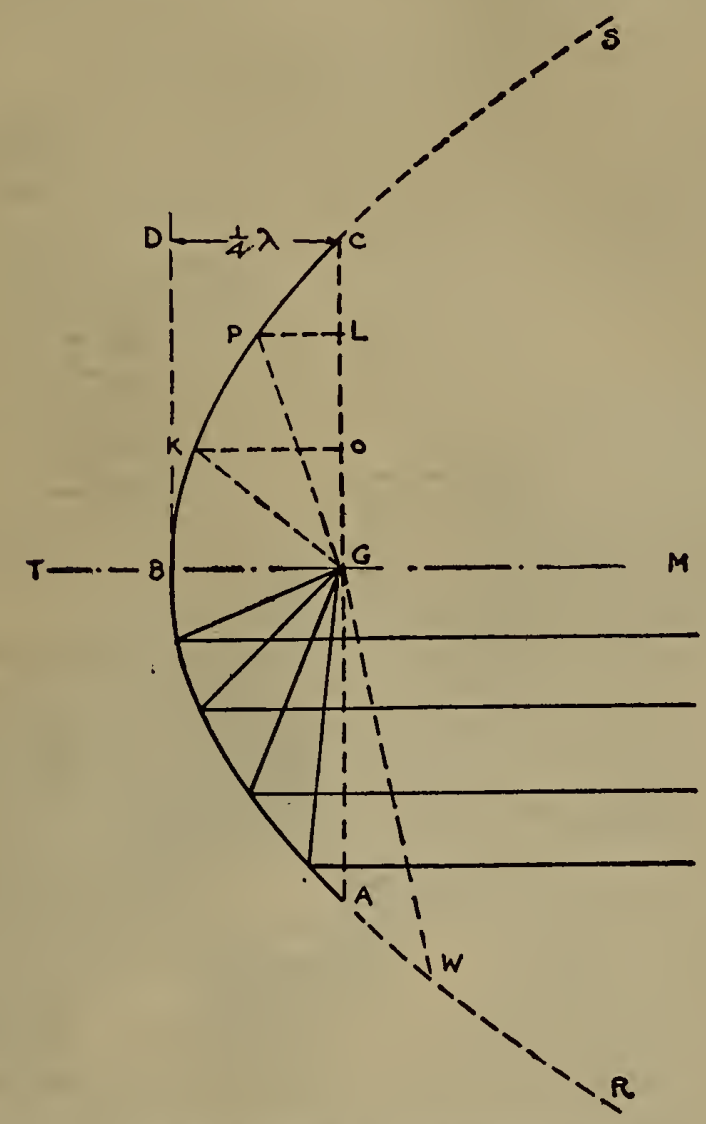

FIG. 5.-Principle of reflection of waves from a parabolic reflector.

over a small angle in the direction $G M$. By extending the parabola, as shown at $S$ and $R$ (increasing the aperture), this side leakage is reduced, thus giving a narrower beam. The type of reflector illustrated was built for experimental use, and while it gives the dimensional values and electrical characteristics the general mechanical design should be made more rugged for practical use. 


\section{RECEIVING APPARATUS.}

In order to study the radiation characteristics of the transmitting system at short distances, the apparatus shown in Figure 6 was employed. This consisted of a single loop of wire about $78.8 \mathrm{~cm}$ ( $3 \mathrm{I}$ inches) in diameter. A two-plate tuning condenser having a maximum capacity of approximately 20 micromicrofarads was inserted in series with this loop. The terminals of the loop were connected to a 5-ohm thermocouple, the output of the couple being connected to a galvanometer having a o-roo microampere scale. At distances up to $52 \mathrm{~m}$ ( 170 feet) from the reflector a full scale deflection on this galvanometer was obtained when the

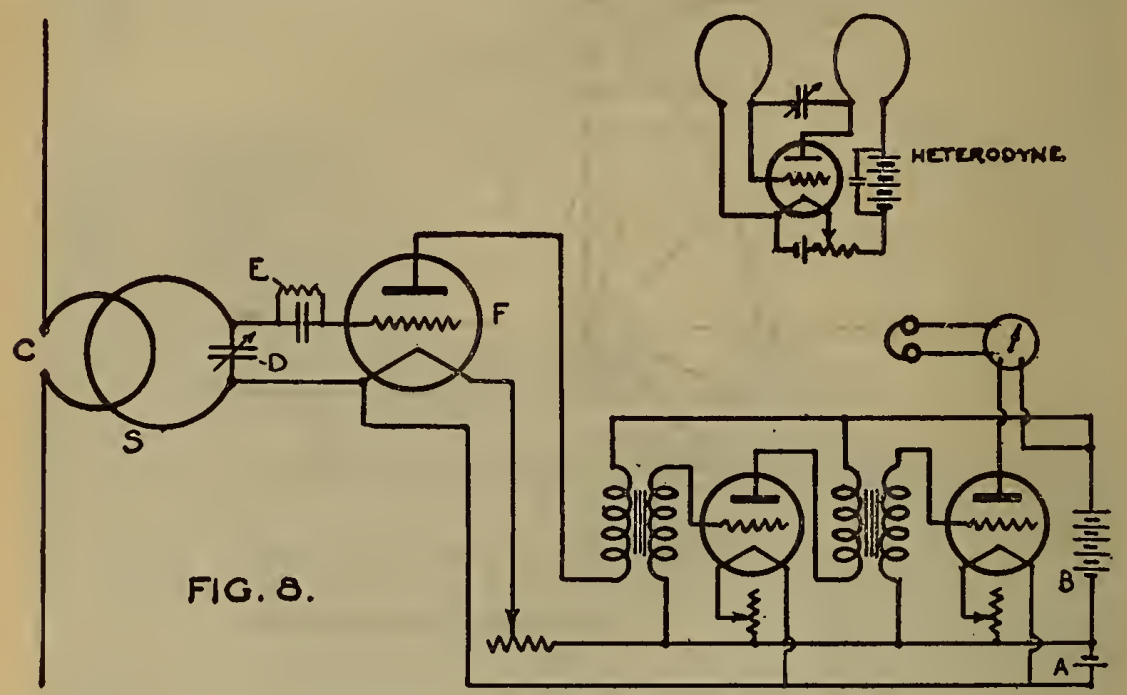

FIG. 8.-Circuit of Io-meter receiving set.

apparatus was adjusted for maximum radiation. This apparatus was used in obtaining most of the polar curves discussed further on in this paper.

For receiving signals at greater distances than $45.8 \mathrm{~m}$ (I 50 feet) a receiving set consisting of a detector and two stages of audiofrequency amplification was used. An external heterodyne was employed when receiving continuous-wave signals. This apparatus is shown in Figure 7 . The secondary of the receiving set, which consisted of a single loop of wire $30.48 \mathrm{~cm}$ ( 12 inches) in diameter, was connected to the grid and filament of the detector tube. A 0.00005 microfarad vernier condenser was shunted across this loop to make tuning possible. A grid leak and grid condenser 
Scientific Papers of the Bureau of Standards, Vol. 19

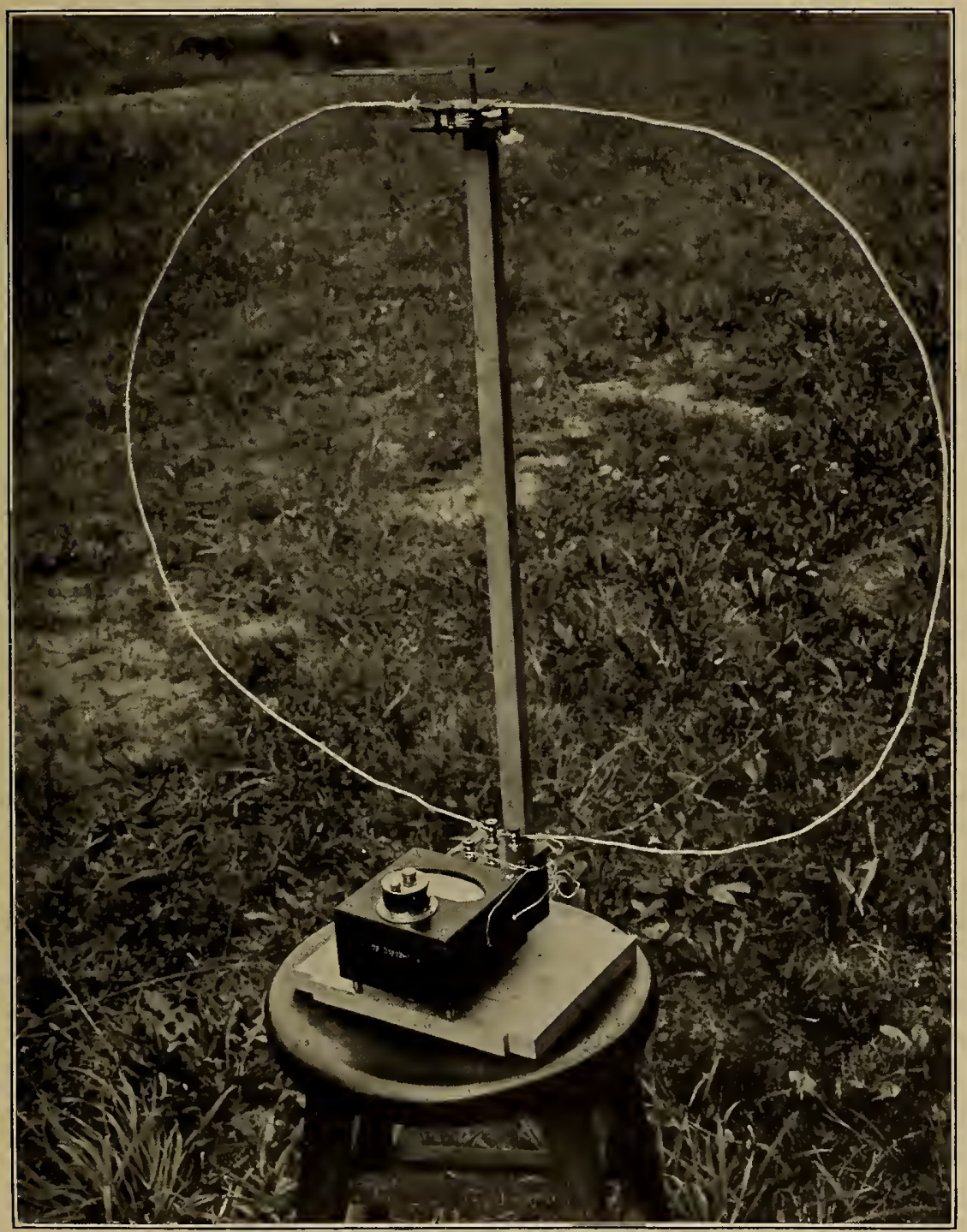

FIG. 6.-Receiving apparatus for studying directive characteristics of radiation from parabolic reflector. 
Scientific Papers of the Bureau of Standards, Vol. 19

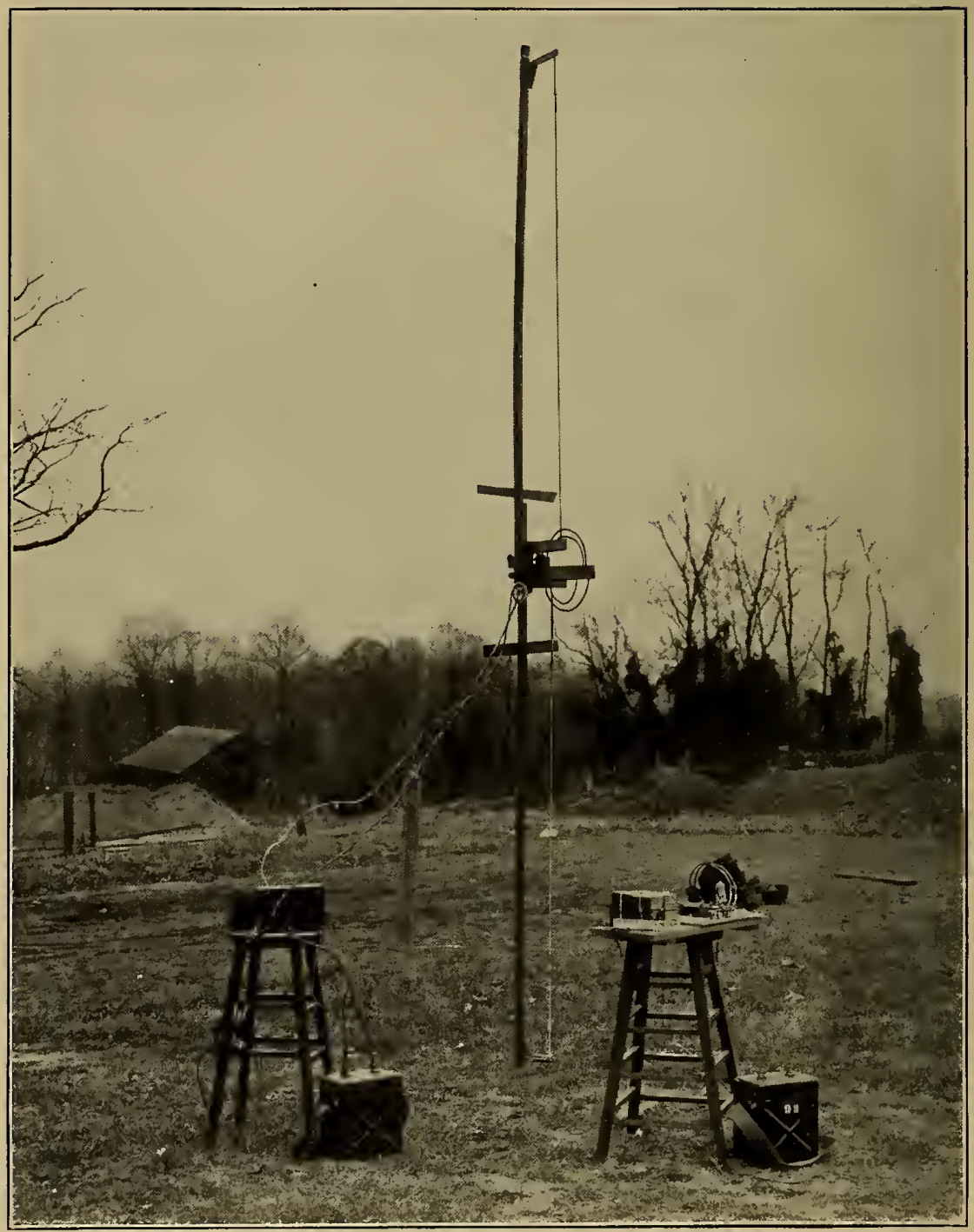

FIG. 7.-Ten-meter receiving set with antenna and heterodyne unit. 
of the usual type was employed. The grid lead was made as short as possible, thus reducing to a minimum the capacity between it and other parts of the circuit. The two stages of audio-frequency amplification were of the usual type.

The antenna (see Fig. 8) used in piciking up the energy at the receiving apparatus was a single wire tuned to the incoming wave frequency and coupled at its center by means of coil $C$ to the secondary coil $S$ of the receiving set. The total length of wire in the antenna (Fig. 8), including the single-turn $30.48 \mathrm{~cm}$ ( $\mathrm{I}$ foot) in diameter in the center, was $4.37 \mathrm{~m}$ ( $\mathrm{I}_{4}$ feet 4 inches). This antenna was made from a single piece of No. I2 B. \& S. gage copper wire. The condenser $D$, grid leak and condenser $E$, and tube $F$ were mounted on the support for $S$ in as close proximity to it as possible. Since this apparatus was attached to the frame supporting $C$ and $S$, it was suspended in the air with it when the signals were being received. (See Fig. 7.)

\section{RESULTS.}

In order to obtain a parabolic reflector of maximum efficiency, experiments were made with various adjustments of the reflector. Two ways of determining the directional characteristics of the radiation from the parabolic reflector are to rotate the reflector while the receiving set remains stationary, or to hold the reflector in a given position and move the receiving set around it in a circle. The former method was used in this case. The apparatus shown in Figure 6 was used in making these measurements. It was set up $52 \mathrm{~m}$ (I 7o feet) from the reflector and oriented to receive maximum energy. The reflector was rotated through $360^{\circ}$ and scale readings of the receiving galvanometer taken for every $10^{\circ}$ position of the reflector. (Figs. 9 to ${ }_{5}$ show the data, in polar curve form, taken in this way.)

Figure 9 shows the radiation characteristic for the parabolic reflector before proper adjustments of the lengths of the reflecting wires had been made; that is, they were not all of the same length. It will be seen from the width of the curve that the reflection was imperfect. Leakage through the rear is indicated by the shaded portion.

Figure Io illustrates the effect obtained after all the wires were made the same in length and when the wave length of the source was made slightly shorter than ro $\mathrm{m}$. This adjustment resulted in throwing all the reflecting wires out of tune. It will 

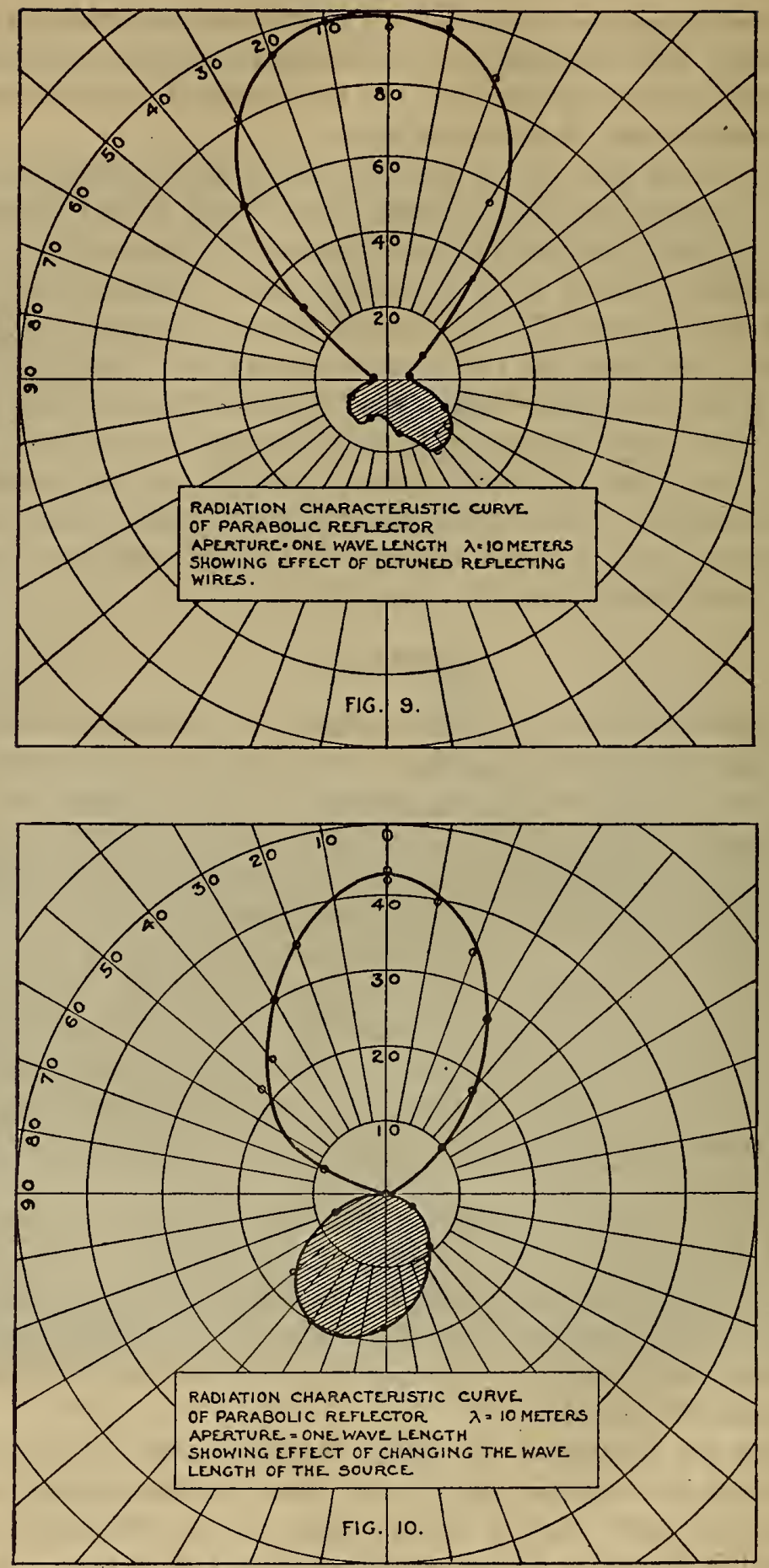
be seen that the radiation through the rear of the reflector was about equal to half that along the true line of reflection.

An effort was next made to obtain the best possible reflection. From the data in Figure 9 it was learned that the proper length of the reflecting wires must be determined while they are in position on the parabolic frame, as the capacity effect of the neighboring wires makes necessary a slightly shorter length of wire for resonance than when they are isolated. After varying the lengths of all the reflecting wires simultaneously and taking

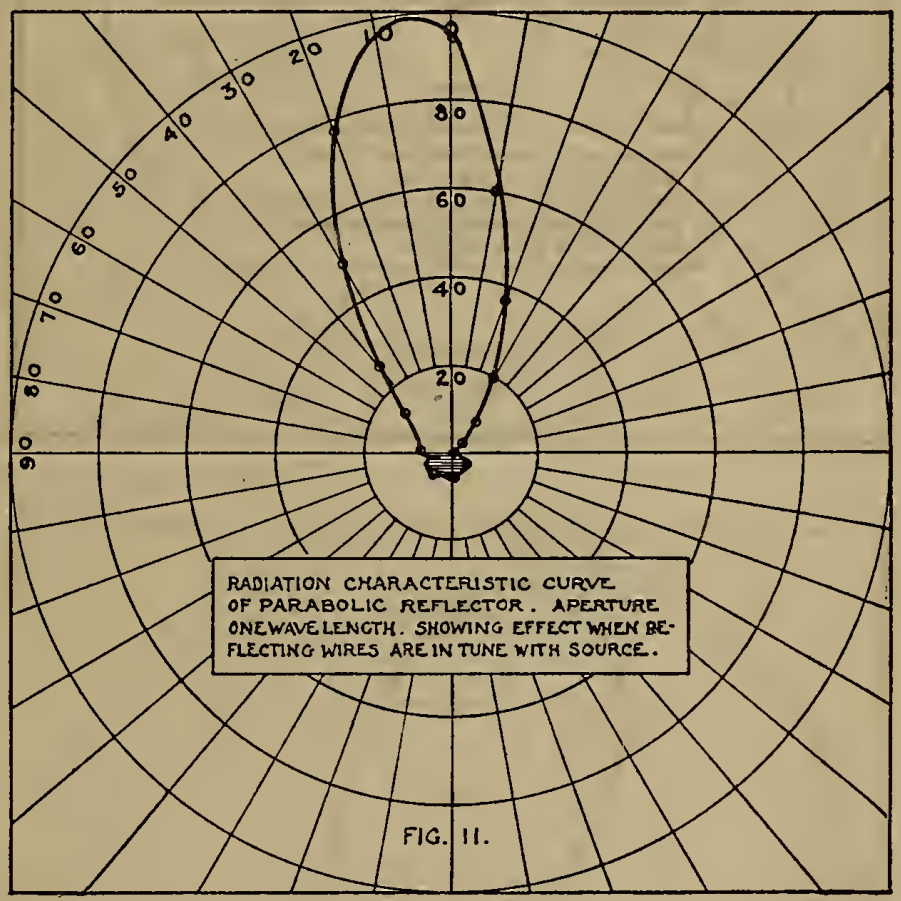

data, one set of data shown in Figure I I was obtained, which indicated that the correct length of the 40 reflecting wires spaced $30.48 \mathrm{~cm}$ (I foot) apart was $4.39 \mathrm{~m}$ (I 4 feet 5 inches). It will be noted that the curve is much sharper, and very little radiation was taking place through the rear of the reflector.

The above data were taken with a reflector having an aperture equal to one wave length. A test was next made with an aperture of 1.5 wave lengths. To do this the parabolic frame was extended and Io tuned wires were suspended, $30.48 \mathrm{~cm}$ (I foot) apart, on each extension. 

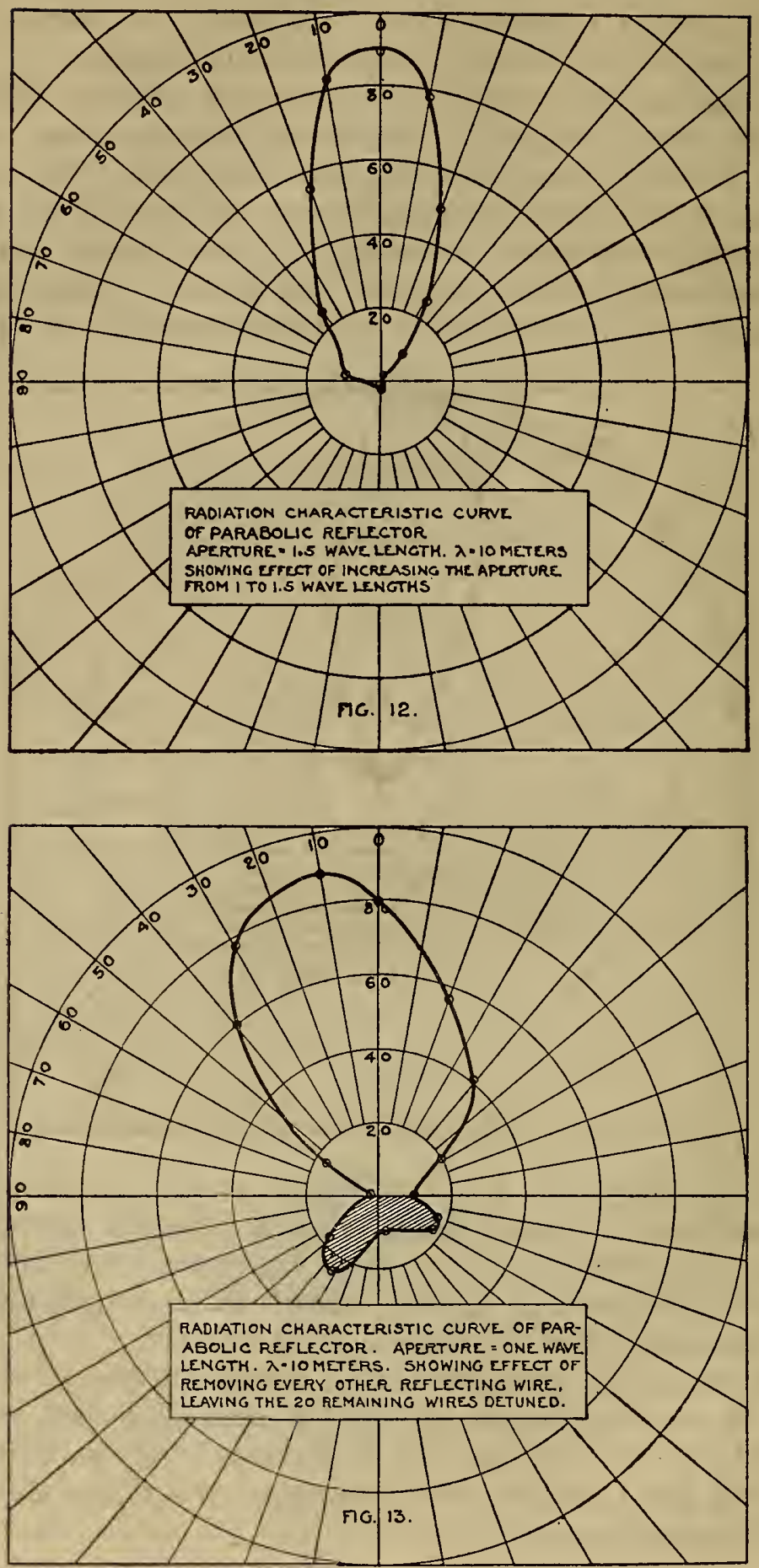
Figure 12 shows the data taken under this condition. It will be seen that there is practically no leakage in the rear of the reflector, and that the reflected wave is in a slightly narrower beam.

Figure $\mathrm{r}_{3}$ illustrates the effect produced when every other reflecting wire is removed; that is, when the number of these wires is reduced from 40 to 20 . Very poor reflection was obtained, and the leakage in the rear was considerable. This was probably caused by the fact that in removing every other wire the remain-

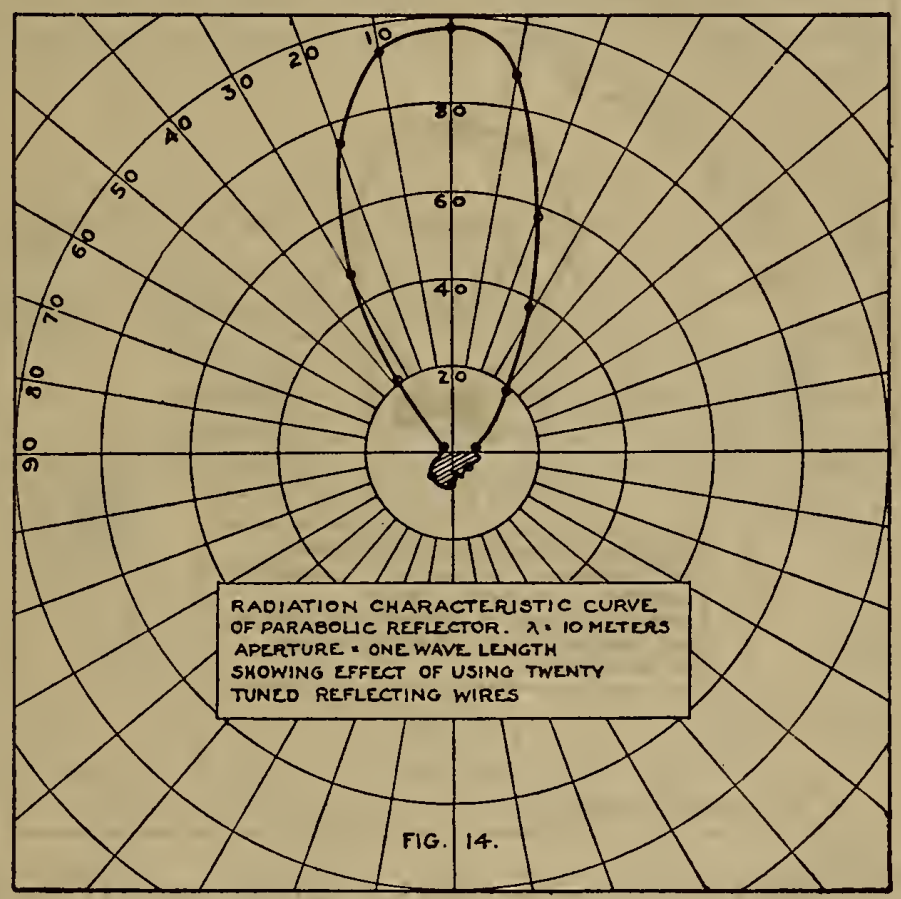

ing wires were detuned, since the capacity of neighboring wires had been removed. This resulted in poor directive radiation, as would be expected.

Figure 14 shows the effect after the 20 reflecting wires had been retuned by increasing their length from $4.39 \mathrm{~m}$ ( $\mathrm{I}_{4}$ feet 5 inches) to $4.47 \mathrm{~m}$ ( 14 feet 8 inches). By comparison with the 40 -wire reflector curve (Fig. II) it will be seen that a slightly sharper beam is obtained by doubling the number of wires.

In order to determine the effect of leakage through an opening in the parabola, the ro reflecting wires nearest the vertex of the parabola were removed. Figure ${ }_{5} 5$ shows the results. It will 
be seen that the reflected beam is very broad, and that there is considerable leakage through the rear. The maximum reflection obtained under this condition was much less in magnitude as would be expected.

From a study of these polar curves it will be seen that certain requirements must be met before efficient directive transmission is obtained. These are: (I) The source of the waves to be reflected should be placed exactly at the focus; (2) the reflecting wires

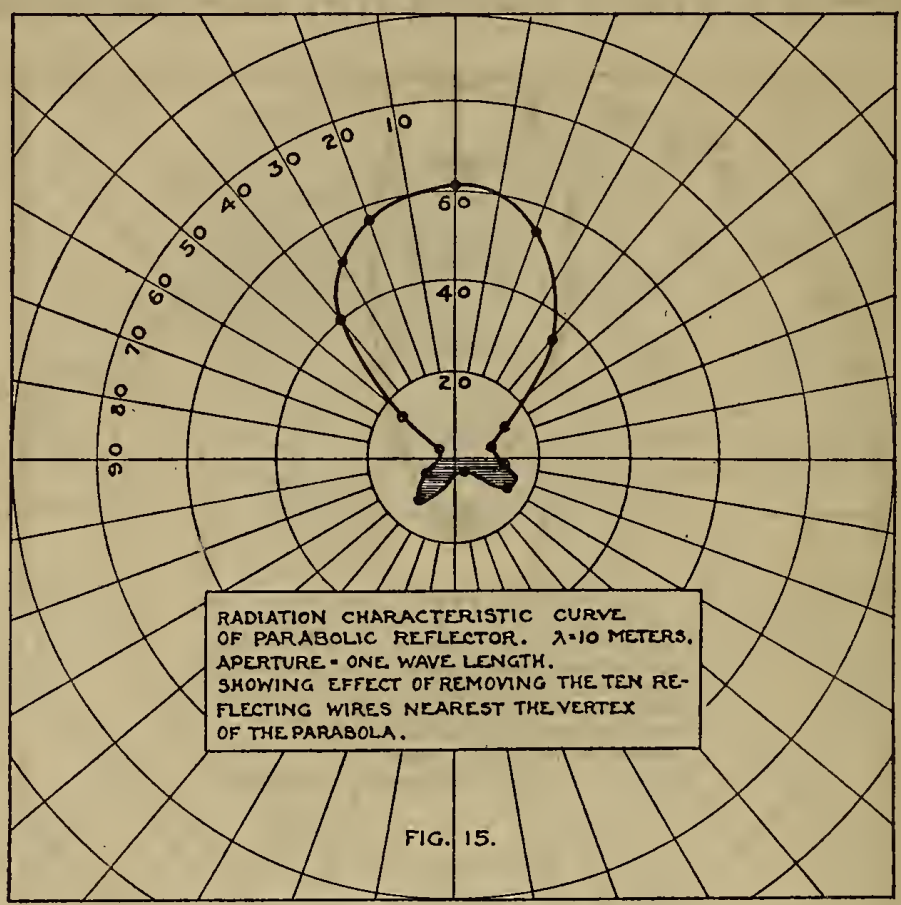

should be tuned to resonance with the source, and (3) the width of the reflected wave front is dependent upon the size of the aperture employed.

It was noted that wave lengths of the order of magnitude used in these tests are absorbed to a very considerable extent by buildings and other metallic structures. An interesting experiment was made, using the receiver shown in Figure 6 . The reflected wave was directed toward a building $5_{50}$ feet away. A threequarter scale deflection on the galvanometer was obtained immediately outside the entrance to this building. When the receiver was taken just inside the door, the deflection dropped to zero, the door being open. Measurements were also made in back 
of the building, the building being between the reflector and receiving loop. The energy received from the reflector was materially decreased whenever any part of the building lay in the path of the wave.

Reception was accomplished at a distance by means of the receiving apparatus shown in Figure 7 . When receiving continuous-wave signals, an external heterodyne was employed. Strong signals were received at a distance of 2 miles, using a singleturn coil antenna 6 inches in diameter. Reception of signals was also accomplished, using this coil antenna coupled to an open oscillator, as shown at $C$ in Figure 8. Very strong signals

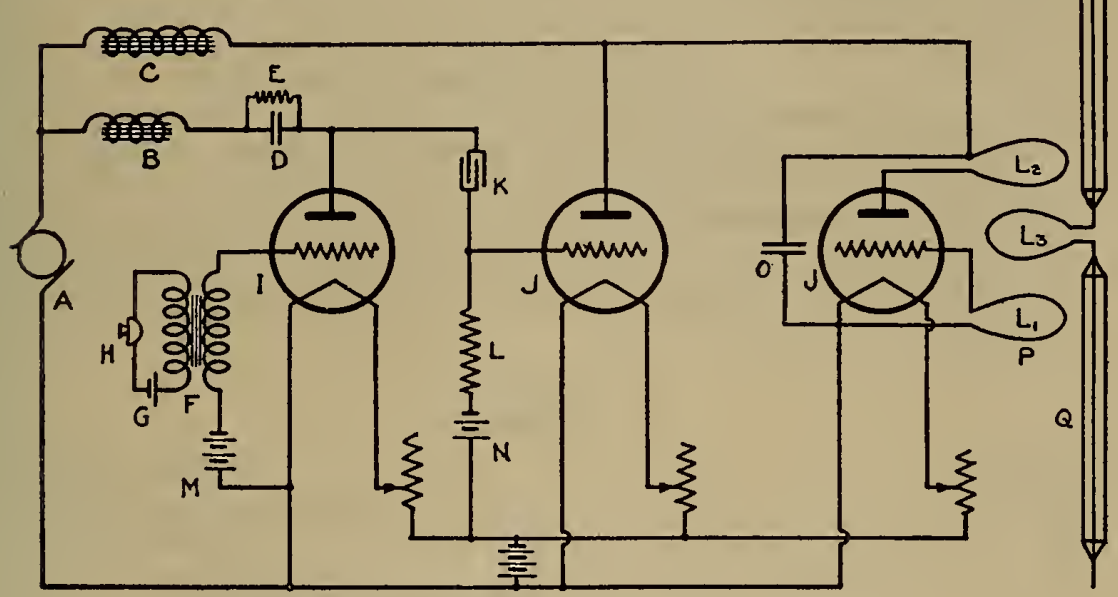

FIG. I6.-Circuit for to-meter radio telephone transmitting set.

were obtained. Reception could have been accomplished at a much greater distance, but lack of time prevented an investigation being made of the maximum range of transmission. The use of reflectors at the receiving station was not attempted. Throughout the time when this antenna was being used for reception no strays were heard. This was probably due to the small dimensions of the antenna employed and the short wave length to which the receiving circuit was tuned.

TELEPHONY.- In order to facilitate tests at a distance, a modulator circuit was attached to the ro $\mathrm{m}$ source, so that telephony was possible. As the reflector was rotated its angular position could be read to the receiving operator. This circuit is shown in Figure 16. Audibility measurements were made in the familiar 
way by means of a shunt circuit across the telephone receivers. A reading was taken when the signal strength had been reduced to a just understandable point.

CoNCLUSICN.--In conclusion it may be said that directive radio comraunication on short wave lengths, employing the type of apparatus described, has been found to be practicable and to merit much more comprehensive investigation and use. The authors are indebted to R. S. Ould for helpful suggestions in the preparation of this paper.

WASHINGTON, January 9, 1923. 